\title{
Cellular immune activity biomarker neopterin is associated hyperlipidemia: results from a large population-based study
}

\author{
Shu-Chun Chuang ${ }^{1,2^{*}}$ (D), Heiner Boeing ${ }^{3}$, Stein Emil Vollset ${ }^{4,5}$, Øivind Midttun ${ }^{6}$, Per Magne Ueland ${ }^{7,8}$, \\ Bas Bueno-de-Mesquita 2,9,10,11 , Martin Lajous ${ }^{12,13,14}$, Guy Fagherazzi ${ }^{12,13,14}$, Marie-Christine Boutron-Ruault ${ }^{12,13,14}$, \\ Rudolf Kaaks ${ }^{15}$, Tilman Küehn ${ }^{15}$, Tobias Pischon ${ }^{16}$, Dagmar Drogan ${ }^{17}$, Anne Tjønneland ${ }^{18}$, Kim Overvad ${ }^{19}$, \\ J Ramón Quirós ${ }^{20}$, Antonio Agudo ${ }^{21}$, Esther Molina-Montes 22,23 , Miren Dorronsoro ${ }^{24}$, José María Huerta ${ }^{23,25}$, \\ Aurelio Barricarte ${ }^{26}$, Kay-Tee Khaw ${ }^{27}$, Nicholas J. Wareham ${ }^{28}$, Ruth C. Travis ${ }^{29}$, Antonia Trichopoulou ${ }^{29,30}$, \\ Pagona Lagiou $31,32,33$, Dimitrios Trichopoulos ${ }^{30,32^{\wedge}}$, Giovanna Masala ${ }^{34}$, Claudia Agnoli ${ }^{35}$, Rosario Tumino ${ }^{36}$, \\ Amalia Mattiello $^{37}$, Petra H Peeters ${ }^{2,38}$, Elisabete Weiderpass ${ }^{39,40,41,42}$, Richard Palmqvist ${ }^{43}$, Ingrid Ljuslinder $^{44}$, \\ Marc Gunter ${ }^{2}$, Yunxia Lu ${ }^{2}$, Amanda J. Cross ${ }^{2}$, Elio Riboli ${ }^{2}$, Paolo Vineis ${ }^{2}$ and Krasimira Aleksandrova ${ }^{45}$
}

\begin{abstract}
Background: Increased serum neopterin had been described in older age two decades ago. Neopterin is a biomarker of systemic adaptive immune activation that could be potentially implicated in metabolic syndrome (MetS). Measurements of waist circumference, triglycerides, high-density lipoprotein cholesterol (HDLC), systolic and diastolic blood pressure, glycated hemoglobin as components of MetS definition, and plasma total neopterin concentrations were performed in 594 participants recruited in the European Prospective Investigation into Cancer and Nutrition (EPIC).

Results: Higher total neopterin concentrations were associated with reduced HDLC $(9.7 \%, p<0.01$ for men and $9.2 \%, p<0.01$ for women), whereas no association was observed with the rest of the MetS components as well as with MetS overall (per $10 \mathrm{nmol} / \mathrm{L}: \mathrm{OR}=1.42,95 \% \mathrm{Cl}=0.85-2.39$ for men and $\mathrm{OR}=1.38,95 \% \mathrm{Cl}=0.79-2.43$ ).
\end{abstract}

Conclusions: These data suggest that high total neopterin concentrations are cross-sectionally associated with reduced HDLC, but not with overall MetS.

Keywords: Neopterin, Cell-mediated immunity, Metabolic syndrome

\section{Background}

Neopterin, a biomarker of systemic adaptive immune activation, is synthesized by monocyte-derived macrophages and dendritic cells upon stimulation of interferon-gamma (IFN- $\gamma$ ) and is considered a reliable proxy to assess the rate of IFN- $\gamma$ production [1-4]. The concentrations of

\footnotetext{
* Correspondence: scchuang@nhri.org.tw

Dimitrios Trichopoulos Deceased.

Deceased

'Institute of Population Health Sciences, National Health Research Institutes,

35 Keyan Road, Zhunan, Miaoli County 35053, Taiwan

${ }^{2}$ Department of Epidemiology and Biostatistics, School of Public Health,

Imperial College London, London, UK

Full list of author information is available at the end of the article
}

neopterin increase with the dose of interferon, thereby help to monitor the activity of INF- $\gamma$ inducible inflammation. Thus, the measurement of neopterin concentrations in body fluids provides information about activation of T-helper cell derived systemic adaptive immune activation [5]. As high neopterin is associated with increased production of reactive oxygen species, neopterin can also be regarded as an indicator for oxidative stress due to immune activation [6].

Neopterin has been used clinically in the assessment of bacterial and viral infections, autoimmune diseases, and malignant conditions [7]. Increased blood neopterin concentrations had been described in older age 
$[8,9]$ and have been positively related to aging-related chronic disorders, including metabolic syndrome (MetS) [3], cancer, cardio-vascular disease, as well as overall mortality [2-4, 10-13].

An emerging field of research - immunometabolism recognizes the existence of an interplay between immunity, inflammation, and impaired metabolism [14]. Central to this theory, inflammation and immune activation are involved in the development of obesity, insulin resistance and potentially also in MetS [14-16]. Despite biological plausibility, only a few epidemiological studies have explored the relation between neopterin and selected metabolic factors. In a study of 3946 patients with acute coronary syndrome, higher plasma concentrations of neopterin were associated with older age, a prior history of hypertension or diabetes, lower low-density lipoprotein cholesterol levels, and higher high-sensitivity C-reactive protein (hsCRP) levels [17]. In another study among 592 patients with high prevalence of MetS, plasma neopterin concentrations were correlated, though weakly, with abdominal obesity, high-density lipoprotein cholesterol (HDLC), and insulin resistance [2]. Similarly, a weak correlation between neopterin and abdominal obesity was reported in another patient cohort of 477 middle-aged and older white individuals at high risk for type 2 diabetes and cardiovascular disease [18].

It remains unclear whether the potential association of neopterin with MetS and its components, may be independent of age and markers of chronic inflammation such as hsCRP. Such knowledge may provide important insights into the potential link between immune activation and impaired metabolism. Therefore, the aim of the study was to investigate the association of plasma total neopterin concentrations with MetS and its components in the European Prospective Investigation into Cancer and Nutrition (EPIC) cohort.

\section{Results}

Overall, the geometric mean of total neopterin concentrations in the study population were 18.74 (standard deviations, SD: 1.50) for men and 18.63 (SD: 1.40) for women. Table 1 shows the characteristics of the study population.

Table 2 presents the Spearman's partial correlation coefficients of clinical markers of MetS and total neopterin concentrations. Total neopterin was inversely correlated with pyridoxal 5 '-phosphate (PLP) and HDLC but positively correlated with hsCRP.

High total neopterin concentrations were associated with lower HDLC, but not with other components of MetS (Table 3). After mutual adjustment, the mean total neopterin concentrations remained different according to HDLC categories ( $p<0.01$ for both men and women). Figure 1 shows the adjusted means and $95 \% \mathrm{CI}$ of total neopterin by increasing number of MetS components. The average differences per component was $4.0 \%\left(P_{\text {difference }}=0.07\right)$ for men and $0.8 \%\left(P_{\text {difference }}=0.64\right)$ for women.

In our study, increased total neopterin was associated with reduced HDLC (OR per $10 \mathrm{nmol} / \mathrm{L}=2.22$, $95 \% \mathrm{CI}=1.24-3.97$ for $\mathrm{men}$ and $\mathrm{OR}$ per $10 \mathrm{nmol} / \mathrm{L}=2.82$, $95 \% \mathrm{CI}=1.68-4.73$ for women), and these associations were independent of PLP (Table 4). Further adjustment for hsCRP did not change the results (OR per $10 \mathrm{nmol} /$ $\mathrm{L}=2.14,95 \% \mathrm{CI}=1.17-3.91$ for men and $\mathrm{OR}$ per 10 $\mathrm{nmol} / \mathrm{L}=2.70,95 \% \mathrm{CI}=1.58-4.61$, data not shown). Increased plasma total neopterin was not associated with overall MetS, defined as presence of any three of the MetS components $(\mathrm{OR}$ per $10 \mathrm{nmol} / \mathrm{L}=1.42,95 \% \mathrm{CI}=0.85-2.39$ for men and $\mathrm{OR}$ per $10 \mathrm{nmol} / \mathrm{L}=1.38,95 \% \mathrm{CI}=0.79-2.43$ for women).

\section{Discussion}

In this study, high total neopterin concentrations were associated with reduced HDLC, but not with overall MetS. These data indicate that immune activation may be related to lipid changes; however, the cross-sectional nature of the study does not provide sufficient information for interpreting the direction of these relations.

Previously only three studies investigated the association of neopterin concentrations with clinical markers of MetS; however, these studies were conducted in participants with underlying diseases, including cardiovascular disease, type 2 diabetes and MetS $[2,18,19]$. In agreement with the study of Oxengrug et al. 2011 [2], we observed an inverse association of total neopterin with HDLC.

Our data suggest an inverse association of total neopterin with HDLC, as well as low-density lipoprotein cholesterol and total cholesterol. This association was independent of age, sex, EPIC study center and smoking status as well as PLP and hsCRP levels. Similar findings have been reported, in patients with HIV infection [20], cardiovascular diseases [4] and MetS [2]. HDLC helps to remove excess cholesterol from peripheral tissue and transport it to the liver for excretion. The functions of HDL include anti-inflammatory and anti-oxidant activities [21]. If the function is impaired, cholesterol accumulates in peripheral tissue and causes inflammation and atherosclerosis. Despite the concept of HDL dysfunction evolved over the last decades, little is known on factors that underline possible alterations between functional and dysfunctional HDL. Recently, immunity was suggested as one of the main pathophysiological pathways of HDLC functionality via modulating cholesterol content in immune cells [22]. It has been shown that inhibition of cholesterol efflux mechanisms in macrophages promotes an inflammatory phenotype in these cells [23]. The raised neopterin levels may indicate activated immune response in individuals 
Table 1 Characteristics of the study population

\begin{tabular}{|c|c|c|c|c|c|c|}
\hline & \multicolumn{2}{|l|}{ Total } & \multicolumn{2}{|l|}{ Men } & \multicolumn{2}{|c|}{ Women } \\
\hline & $\mathrm{N}$ & $\%$ & $\mathrm{~N}$ & $\%$ & $\mathrm{~N}$ & $\%$ \\
\hline Age, mean (SD) & 57.59 & $(8.24)$ & 58.24 & $(7.81)$ & 57.04 & $(8.55)$ \\
\hline \multicolumn{7}{|l|}{ Education } \\
\hline None or primary school completed & 247 & 42 & 118 & 44 & 129 & 40 \\
\hline Technical/professional school & 123 & 21 & 58 & 21 & 65 & 20 \\
\hline Secondary school & 83 & 14 & 16 & 6 & 67 & 21 \\
\hline Longer education & 119 & 20 & 66 & 24 & 53 & 16 \\
\hline Not specified & 22 & 4 & 12 & 4 & 10 & 3 \\
\hline \multicolumn{7}{|l|}{ Smoking status } \\
\hline Never & 273 & 46 & 80 & 30 & 193 & 60 \\
\hline Former & 196 & 33 & 125 & 46 & 71 & 22 \\
\hline Current & 118 & 20 & 61 & 23 & 57 & 18 \\
\hline Unknown & 7 & 1 & 4 & 1 & 3 & 1 \\
\hline \multicolumn{7}{|l|}{ Physical activity } \\
\hline Low & 102 & 17 & 60 & 22 & 42 & 13 \\
\hline Medium & 121 & 20 & 62 & 23 & 59 & 18 \\
\hline High & 139 & 23 & 53 & 20 & 86 & 27 \\
\hline Very high & 205 & 35 & 77 & 29 & 128 & 40 \\
\hline Missing & 27 & 5 & 18 & 7 & 9 & 3 \\
\hline Waist circumference, cm, mean (SD) & 87.22 & $(11.83)$ & 94.37 & $(9.33)$ & 81.41 & $(10.38)$ \\
\hline Tryglicerides, mmol/L, mean (SD) & 1.19 & $(0.94)$ & 1.29 & $(1.07)$ & 1.10 & $(0.80)$ \\
\hline High-density lipoprotein cholesterol, mmol/L, mean (SD) & 1.43 & $(0.39)$ & 1.29 & $(0.36)$ & 1.54 & $(0.38)$ \\
\hline Systolic blood pressure, mmHg, mean (SD) & 128.93 & $(16.77)$ & 131.41 & $(15.70)$ & 127.14 & $(17.31)$ \\
\hline Diastolic blood pressure, mmHg, mean (SD) & 79.72 & $(9.84)$ & 81.94 & $(9.76)$ & 78.12 & $(9.60)$ \\
\hline HbA1c (\%), mean (SD) & 5.74 & $(0.61)$ & 5.77 & $(0.63)$ & 5.71 & $(0.60)$ \\
\hline Nopterin (nmol/L), mean (SD) & 20.07 & $(7.90)$ & 20.39 & (8.37) & 19.81 & $(7.49)$ \\
\hline
\end{tabular}

with low HDL cholesterol levels. Neopterin had been associated with cardiovascular events $[4,24,25]$, suggesting a potential involvement of adaptive immunity and inflammation in modulating the association between cholesterol metabolism and cardio-metabolic risk. From a practical perspective, measurement of neopterin in addition to HDLC may aid in identifying HDL anti-inflammatory/ proinflammatory function and could likely yield important additional information beyond that available from simple measurement of HDLC in an individual. However, future studies are needed in order to evaluate potential practical implication of these findings. Of note, despite the association with HDLC, we observed no association with previous diagnosis of hyperlipidemia (data not shown). This can be partly explained by the observation that total neopterin concentrations were lower in those who used lipid-lowering drugs [4].

Previous studies have reported positive associations between neopterin and waist circumference [2, 10, 18, 26, 27]. In one of these; however, such association disappeared after adjustment for other metabolic biomarkers [18]. Thewissen et al. (2011) reported that the association between abdominal fat and neopterin - considered a marker of adaptive immune activation - was mediated, by elevations in hsCRP and other immune activation markers [18]. They hypothesized that it is not merely an increased mass of adipose tissue that directly leads to attenuation of insulin action, but rather adipose tissue inflammation mediated by activated immune system in obese individuals that leads to insulin resistance. In our study, we only observed a non-statistically significant marginal association between abdominal obesity and total neopterin concentrations. Further prospective studies are needed to test this hypothesis.

Similarly to a previous report [4], we found no association between total neopterin concentrations and triglycerides (TG). There was no association between total neopterin concentrations and measured systolic or diastolic blood pressure (BP), including pre-defined cutoffs for hypertension. There have been reports suggesting 
Table 2 Spearman partial correlation coefficients $(r)^{1}$ between total neopterin, pyridoxal $5^{\prime}$-phosphate (PLP) and markers of metabolic factors

\begin{tabular}{|c|c|c|c|c|c|c|c|c|c|c|c|c|c|c|c|c|}
\hline & \multicolumn{2}{|c|}{$\mathrm{PLP}, \mu \mathrm{mol} / \mathrm{L}$} & \multicolumn{2}{|c|}{ Waist circumference, $\mathrm{cm}$} & \multicolumn{2}{|c|}{$\begin{array}{l}\text { Tryglicerides, } \\
\mathrm{mmol} / \mathrm{L}\end{array}$} & \multicolumn{2}{|c|}{$\begin{array}{l}\text { High-density lipoprotein } \\
\text { cholesterol, } \mathrm{mmol} / \mathrm{L}\end{array}$} & \multicolumn{2}{|c|}{$\begin{array}{l}\text { Systolic blood } \\
\text { pressure }(\mathrm{mmHg})\end{array}$} & \multicolumn{2}{|c|}{$\begin{array}{l}\text { Diastolic blood } \\
\text { pressure, } \mathrm{mmHg}\end{array}$} & \multicolumn{2}{|c|}{$\mathrm{HbA1c}(\%)$} & \multicolumn{2}{|c|}{$\overline{h s C R P, ~ m g / L ~}$} \\
\hline & $\rho$ & $p$ & $\rho$ & $p$ & $\rho$ & $\mathrm{p}$ & $\rho$ & $p$ & $\rho$ & $p$ & $\rho$ & $p$ & $\rho$ & $p$ & $\rho$ & $\mathrm{p}$ \\
\hline & Men & & & & & & & & & & & & & & & \\
\hline Neopterin, nmol/L & -0.13 & 0.04 & 0.24 & $<0.01$ & -0.07 & 0.29 & -0.18 & 0.01 & 0.09 & 0.22 & 0.16 & 0.04 & 0.01 & 0.91 & 0.18 & 0.01 \\
\hline $\mathrm{PLP}, \mu \mathrm{mol} / \mathrm{L}$ & & & 0.08 & 0.24 & -0.04 & 0.55 & 0.21 & $<0.01$ & -0.18 & 0.07 & -0.05 & 0.57 & -0.09 & 0.35 & -0.11 & 0.11 \\
\hline Waist circumference, $\mathrm{cm}$ & & & & & 0.26 & $<0.01$ & -0.23 & $<0.01$ & 0.26 & $<0.01$ & 0.28 & $<0.01$ & 0.12 & 0.15 & 0.21 & 0.02 \\
\hline Tryglicerides, mmol/L & & & & & & & -0.35 & $<0.01$ & 0.15 & 0.04 & 0.24 & 0.00 & 0.06 & 0.50 & 0.02 & 0.79 \\
\hline $\begin{array}{l}\text { High-density lipoprotein } \\
\text { cholesterol, mmol/L }\end{array}$ & & & & & & & & & -0.18 & 0.01 & -0.17 & 0.02 & 0.04 & 0.58 & -0.17 & 0.01 \\
\hline Systolic blood pressure, $\mathrm{mmHg}$ & & & & & & & & & & & 0.66 & $<0.01$ & 0.11 & 0.27 & 0.08 & 0.29 \\
\hline Diastolic blood pressure, $\mathrm{mmHg}$ & & & & & & & & & & & & & -0.01 & 0.89 & 0.07 & 0.36 \\
\hline \multirow[t]{2}{*}{$\mathrm{HbA1c}(\%)$} & & & & & & & & & & & & & & & 0.07 & 0.45 \\
\hline & Wome & & & & & & & & & & & & & & & \\
\hline Neopterin, nmol/L & -0.15 & 0.01 & 0.10 & 0.09 & 0.02 & 0.68 & -0.24 & $<0.01$ & -0.05 & 0.43 & -0.07 & 0.28 & -0.11 & 0.23 & 0.15 & 0.01 \\
\hline $\mathrm{PLP}, \mu \mathrm{mol} / \mathrm{L}$ & & & -0.06 & 0.29 & -0.08 & 0.17 & 0.14 & 0.02 & -0.08 & 0.44 & -0.03 & 0.78 & 0.25 & 0.01 & -0.20 & $<0.01$ \\
\hline Waist circumference, $\mathrm{cm}$ & & & & & 0.31 & $<0.01$ & -0.28 & $<0.01$ & 0.25 & $<0.01$ & 0.21 & $<0.01$ & 0.14 & 0.15 & 0.27 & $<0.01$ \\
\hline Tryglicerides, mmol/L & & & & & & & -0.35 & $<0.01$ & 0.16 & 0.01 & 0.13 & 0.03 & 0.07 & 0.50 & 0.15 & 0.12 \\
\hline $\begin{array}{l}\text { High-density lipoprotein } \\
\text { cholesterol, mmol/L }\end{array}$ & & & & & & & & & -0.04 & 0.48 & 0.01 & 0.89 & 0.14 & 0.12 & -0.09 & 0.12 \\
\hline Systolic blood pressure, $\mathrm{mmHg}$ & & & & & & & & & & & 0.64 & $<0.01$ & 0.01 & 0.95 & 0.19 & $<0.01$ \\
\hline Diastolic blood pressure, $\mathrm{mmHg}$ & & & & & & & & & & & & & 0.03 & 0.76 & 0.04 & 0.56 \\
\hline $\mathrm{HbA} 1 \mathrm{c}(\%)$ & & & & & & & & & & & & & & & 0.00 & 0.99 \\
\hline
\end{tabular}

${ }^{1}$ The partial correlation coefficients were adjusted for age at blood collection (years), sex, EPIC study centers, and smoking status (never, former, current, and unknown)

Abbreviations: $P L P$ pyridoxal 5'-phosphate, WC waist circumference, TG triglycerides, HDLC high-density lipoprotein cholesterol, SBP systolic blood pressure, DBP

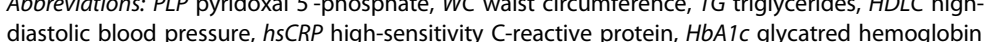


Table 3 Adjusted geometric means and $95 \%$ confidence intervals (95\% Cl) of the means of total neopterin by levels of pyridoxal 5'-phosphate (PLP) and markers of metabolic factors

\begin{tabular}{|c|c|c|c|c|c|c|c|c|c|c|}
\hline \multirow{3}{*}{$\overline{P L P}, \mu \mathrm{mol} / \mathrm{L}$} & \multicolumn{5}{|l|}{ Men } & \multicolumn{5}{|c|}{ Women } \\
\hline & \multirow[t]{2}{*}{$\overline{\text { Mean }^{1}}$} & \multicolumn{2}{|c|}{$95 \% \mathrm{Cl}$} & \multirow[t]{2}{*}{ Difference $^{2}$} & \multirow[t]{2}{*}{$p$} & \multirow[t]{2}{*}{ Mean $^{1}$} & \multicolumn{2}{|c|}{$95 \% \mathrm{Cl}$} & \multirow[t]{2}{*}{ Difference $^{2}$} & \multirow[t]{2}{*}{$p$} \\
\hline & & & & & & & & & & \\
\hline T1 (M: <=28.1 (Median:21.4); F: <=24.5 (Median:19.6)) & 18.09 & 15.67 & 20.89 & & & 15.53 & 13.42 & 17.98 & & \\
\hline T2 (M: 28.1-44.9 (Median:34.7); F: 24.5-38.9 (Median:30.4)) & 17.26 & 14.81 & 20.11 & $-4.7 \%$ & 0.43 & 14.68 & 12.81 & 16.83 & $-7.3 \%$ & 0.11 \\
\hline T3 (M: >44.9 (Median:63.5); F: >38.9 (Median:53.9)) & 16.72 & 14.52 & 19.24 & $-7.9 \%$ & 0.18 & 16.71 & 14.60 & 19.13 & $-13.0 \%$ & 0.01 \\
\hline Per tertile & & & & $-3.9 \%$ & 0.18 & & & & $-6.5 \%$ & 0.01 \\
\hline \multicolumn{11}{|l|}{ Waist circumference $(\mathrm{cm})$} \\
\hline T1 (M: <=91.01 (Median:86.45); F: <=76.77 (Median:71.50)) & 17.26 & 14.90 & 19.98 & & & 16.03 & 13.77 & 18.67 & & \\
\hline T2 (M: 91.01-98.71 (Median:95); F: 76.77-85.51 (Median:80.50)) & 18.93 & 16.37 & 21.89 & $9.2 \%$ & 0.12 & 16.25 & 13.91 & 18.98 & $1.3 \%$ & 0.77 \\
\hline T3 (M: >98.71 (Median:104); F: >85.51 (Median:93)) & 19.17 & 16.44 & 22.37 & $10.5 \%$ & 0.11 & 16.93 & 14.62 & 19.61 & $5.4 \%$ & 0.26 \\
\hline Per tertile & & & & $5.3 \%$ & 0.11 & & & & $2.7 \%$ & 0.27 \\
\hline \multicolumn{11}{|l|}{ Triglyceride $(\mathrm{mmol} / \mathrm{L})$} \\
\hline$<1.7$ & 17.07 & 14.95 & 19.49 & & & 16.24 & 13.94 & 18.92 & & \\
\hline $1.7-3.4$ & 17.09 & 14.62 & 19.99 & $0.2 \%$ & 0.98 & 16.21 & 14.01 & 18.75 & $-3.7 \%$ & 0.53 \\
\hline$>=3.4$ & 21.66 & 16.99 & 27.61 & $23.8 \%$ & 0.03 & 16.29 & 13.94 & 19.03 & $9.3 \%$ & 0.48 \\
\hline Per mmol/L & & & & $5.9 \%$ & 0.03 & & & & $2.3 \%$ & 0.56 \\
\hline \multicolumn{11}{|l|}{ High-density lipoprotein cholesterol (mmol/L) } \\
\hline T1 (M: <=1.10 (median:0.98); F: <=1.33 (median:1.18)) & 19.72 & 17.09 & 22.75 & & & 18.52 & 15.97 & 21.47 & & \\
\hline T2 (M: 1.10-1.34 (median:1.21); F: 1.33-1.62 (median:1.49)) & 16.52 & 14.33 & 19.04 & $-17.7 \%$ & 0.00 & 15.73 & 13.62 & 18.15 & $-16.4 \%$ & 0.00 \\
\hline T3 (M: >1.34 (median:1.60); F: >1.62 (median:1.87)) & 16.29 & 14.15 & 18.75 & $-19.1 \%$ & 0.00 & 15.44 & 13.36 & 17.85 & $-18.2 \%$ & $<0.01$ \\
\hline Per tertile & & & & $-9.7 \%$ & 0.00 & & & & $-9.2 \%$ & $<0.01$ \\
\hline \multicolumn{11}{|l|}{ Low-density lipoprotein cholesterol (mmol/L) } \\
\hline Q1 (M: <3.88 (median:3.37); F: <3.88 (median:3.34)) & 18.96 & 16.39 & 21.94 & & & 16.65 & 14.49 & 19.14 & & \\
\hline Q2 (M: 3.88-4.70 (median:4.25); F: 3.88-4.90 (median:4.34)) & 16.79 & 14.64 & 19.27 & $-12.2 \%$ & 0.03 & 15.60 & 13.59 & 17.92 & $-6.5 \%$ & 0.17 \\
\hline Q3 (M: $\geq 4.70$ (median:5.29); F: $\geq 4.90$ (median:5.55)) & 16.63 & 14.37 & 19.26 & $-13.1 \%$ & 0.03 & 14.76 & 12.83 & 16.98 & $-12.1 \%$ & 0.02 \\
\hline Per tertile & & & & $-6.6 \%$ & 0.03 & & & & $-6.0 \%$ & 0.02 \\
\hline \multicolumn{11}{|l|}{ Total cholesterol (mmol/L) } \\
\hline Q1 (M: <5.74 (median:5.16); F: <5.94 (median:5.29)) & 19.19 & 16.58 & 22.21 & & & 16.61 & 14.43 & 19.12 & & \\
\hline Q2 (M: 5.74-6.59 (median:6.17); F: 5.94-6.99 (median:6.49)) & 16.61 & 14.48 & 19.06 & $-14.4 \%$ & 0.01 & 15.47 & 13.46 & 17.79 & $-7.1 \%$ & 0.15 \\
\hline Q3 (M: $\geq 6.59$ (median:7.32); F: $\geq 6.99$ (median:7.69)) & 16.85 & 14.53 & 19.54 & $-13.0 \%$ & 0.03 & 15.03 & 13.07 & 17.29 & $-10.0 \%$ & 0.05 \\
\hline Per tertile & & & & $-6.8 \%$ & 0.03 & & & & $-4.9 \%$ & 0.05 \\
\hline \multicolumn{11}{|l|}{ Blood pressure } \\
\hline \multicolumn{11}{|l|}{ Systolic BP } \\
\hline$<=123$ & 16.87 & 14.59 & 19.51 & & & 16.20 & 13.68 & 19.18 & & \\
\hline 123-139 & 17.01 & 14.66 & 19.74 & $0.8 \%$ & 0.89 & 16.15 & 13.66 & 19.09 & $-0.3 \%$ & 0.95 \\
\hline$>139$ & 18.14 & 15.50 & 21.24 & $7.3 \%$ & 0.28 & 15.75 & 13.31 & 18.64 & $-2.8 \%$ & 0.61 \\
\hline Per tertile & & & & $3.6 \%$ & 0.27 & & & & $-1.4 \%$ & 0.60 \\
\hline \multicolumn{11}{|l|}{ Diastolic BP } \\
\hline$<=76$ & 16.76 & 14.53 & 19.34 & & & 16.43 & 13.88 & 19.46 & & \\
\hline $76-85$ & 16.93 & 14.59 & 19.65 & $1.0 \%$ & 0.87 & 16.11 & 13.68 & 18.98 & -0.02 & 0.70 \\
\hline$>85$ & 18.50 & 15.85 & 21.60 & $9.9 \%$ & 0.12 & 15.32 & 12.90 & 18.19 & -0.07 & 0.19 \\
\hline Per tertile & & & & $4.9 \%$ & 0.11 & & & & $-3.5 \%$ & 0.19 \\
\hline
\end{tabular}


Table 3 Adjusted geometric means and $95 \%$ confidence intervals (95\% Cl) of the means of total neopterin by levels of pyridoxal 5'-phosphate (PLP) and markers of metabolic factors (Continued)

Systolic BP $\geq 130$ or diastolic $B P \geq 85$ mmHg or diagnosis for hypertension
No
Yes

${ }^{1}$ The means were calculated by exponentiating the natural-log transformed means, which were estimated from multiple linear regression and adjusted for age at blood collection (years), sex, country, education (none or primary school completed, technical or professional school, secondary school, above secondary school, and not specified), smoking status (never, former, current, and unknown), and physical activity (low, medium, high, very high, missing)

2.The differences compared to the first category of each variable

Abbreviations: $T$ tertile, PLP pyridoxal 5'-phosphate, BP blood pressure, HbA1C glycated hemoglobin

that neopterin could be a predictive marker for cardiovascular events $[4,19,24,25,28-31]$, including an elevated diastolic BP [29, 32]. However, its associations with hypertension (or BP) have been inconsistent across studies. In this context, our results might suggest that although hypertension is an important component of cardiovascular diseases, it might not be directly associated with inflammation or IFN $-\gamma$ mediated inflammation.

Previous studies have also reported that neopterin concentrations were positively associated with glucose

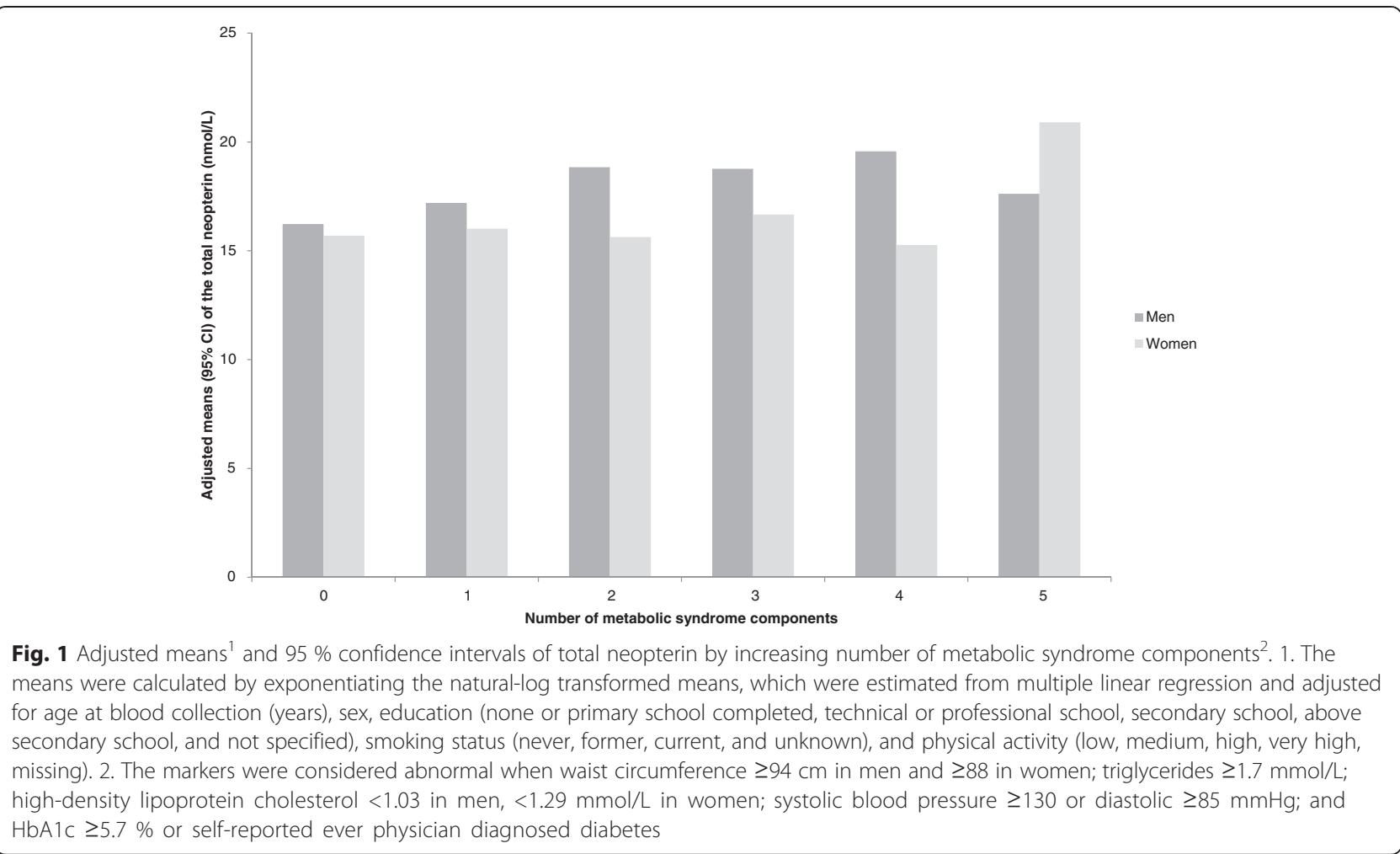


Table 4 Association between total neopterin and metabolic syndrome (MetS) ${ }^{1}$ and its components

\begin{tabular}{|c|c|c|c|c|c|c|c|c|c|c|c|c|c|c|c|c|c|}
\hline \multirow{4}{*}{$\begin{array}{l}\text { MetS components } \\
\text { Waist circumference } \geq 94 \mathrm{~cm} \text { in men }\end{array}$} & \multicolumn{17}{|c|}{ Total Neopterin, nmol/L, Men } \\
\hline & \multicolumn{3}{|l|}{$\mathrm{T} 1^{2}$} & \multicolumn{5}{|l|}{$\mathrm{T} 2^{2}$} & \multicolumn{5}{|l|}{$\mathrm{T}^{2}$} & \multicolumn{4}{|c|}{ Per $10 \mathrm{nmol} / \mathrm{L}$} \\
\hline & \multirow{2}{*}{$\begin{array}{l}\text { Normal } \\
52\end{array}$} & \multirow{2}{*}{$\begin{array}{l}\text { Abnormal } \\
37\end{array}$} & \multirow{2}{*}{$\begin{array}{l}\mathrm{OR}^{4} \\
1.00\end{array}$} & \multirow{2}{*}{$\begin{array}{l}\text { Normal } \\
39\end{array}$} & \multirow{2}{*}{$\begin{array}{l}\text { Abnormal } \\
51\end{array}$} & \multirow{2}{*}{$\begin{array}{l}\mathrm{OR}^{4} \\
2.00\end{array}$} & \multicolumn{2}{|c|}{$95 \% \mathrm{Cl}$} & \multirow{2}{*}{$\begin{array}{l}\text { Normal } \\
31\end{array}$} & \multirow{2}{*}{$\begin{array}{l}\text { Abnormal } \\
49\end{array}$} & \multirow{2}{*}{$\frac{O R^{4}}{2.12}$} & \multicolumn{2}{|c|}{$95 \% \mathrm{Cl}$} & \multirow{2}{*}{$\begin{array}{l}\mathrm{OR}^{4} \\
1.64\end{array}$} & \multicolumn{2}{|c|}{$95 \% \mathrm{Cl}$} & \multirow{2}{*}{$\frac{P_{\text {trend }}}{0.05}$} \\
\hline & & & & & & & $(1.00$ & 3.99) & & & & $(1.01$ & 4.42) & & (0.99, & 2.73) & \\
\hline Triglycerides $\geq 1.7 \mathrm{mmol} / \mathrm{L}$ & 77 & 22 & 1.00 & 67 & 22 & 1.21 & (0.57, & 2.56) & 58 & 22 & 1.14 & $(0.52$ & 2.50) & 1.08 & $(0.64$ & 1.84) & 0.77 \\
\hline High-density lipoprotein cholesterol , <1.03 in men & 90 & 9 & 1.00 & 69 & 21 & 2.75 & $(1.13$ & $6.70)$ & 56 & 24 & 3.71 & $(1.48$, & 9.32) & 2.22 & $(1.24$ & 3.97) & 0.01 \\
\hline $\begin{array}{l}\text { Systolic blood pressure } \geq 130 \text { or diastolic } \geq 85 \mathrm{mmHg} \text { or } \\
\text { diagnosis for hypertension }\end{array}$ & 49 & 50 & 1.00 & 50 & 40 & 0.93 & $(0.43$ & 2.04) & 37 & 43 & 0.82 & $(0.34$ & 1.95) & 0.87 & $(0.48$ & 1.59) & 0.65 \\
\hline $\begin{array}{l}\text { HbA1c } \geq 5.7 \% \text { or self-reported ever physician diagnosed } \\
\text { diabetes }\end{array}$ & 69 & 30 & 1.00 & 53 & 37 & 1.62 & $(0.83$ & 3.15) & 50 & 30 & 1.47 & $(0.72$ & $3.01)$ & 1.28 & $(0.79$ & 2.08) & 0.32 \\
\hline Any three of the above & 86 & 13 & 1.00 & 73 & 18 & 2.02 & $(0.95$ & 4.30) & 61 & 19 & 1.82 & $(0.83$ & 3.99) & 1.42 & $(0.85$ & 2.39) & 0.18 \\
\hline \multirow[t]{3}{*}{ MetS components } & \multicolumn{17}{|c|}{ Total Neopterin, nmol/L, Women } \\
\hline & \multicolumn{3}{|l|}{$\mathrm{T} 1^{2}$} & \multicolumn{5}{|l|}{$\mathrm{T} 2^{2}$} & \multicolumn{5}{|l|}{$\mathrm{T}^{2}$} & \multicolumn{4}{|c|}{ Per $10 \mathrm{nmol} / \mathrm{L}$} \\
\hline & Normal & Abnormal & $O R^{4}$ & Normal & Abnormal & $O R^{4}$ & $\begin{array}{l}95 \% \\
\mathrm{Cl}\end{array}$ & & Normal & Abnormal & $O R^{4}$ & $\begin{array}{l}95 \% \\
\mathrm{Cl}\end{array}$ & & $\mathrm{OR}^{4}$ & $\begin{array}{l}95 \% \\
\mathrm{Cl}\end{array}$ & & $P_{\text {trend }}$ \\
\hline Waist circumference $\geq 80 \mathrm{~cm}$ in women ${ }^{3}$ & 68 & 51 & 1.00 & 49 & 57 & 1.48 & $(0.83$ & 2.61) & 44 & 50 & 1.27 & $(0.70$ & 2.31) & 1.21 & $(0.76$ & 1.94) & 0.43 \\
\hline Triglycerides $\geq 1.7 \mathrm{mmol} / \mathrm{L}$ & 104 & 17 & 1.00 & 90 & 15 & 0.97 & $(0.43$ & 2.16) & 80 & 15 & 0.87 & $(0.38$ & 2.00) & 0.89 & $(0.46$ & $1.73)$ & 0.74 \\
\hline High-density lipoprotein cholesterol, <1.29 mmol/L in women & 102 & 19 & 1.00 & 72 & 34 & 2.90 & $(1.48$ & 5.70) & 58 & 37 & 4.05 & $(2.04$ & 8.03) & 2.82 & $(1.68$, & 4.73) & $<0.01$ \\
\hline $\begin{array}{l}\text { Systolic blood pressure } \geq 130 \text { or diastolic } \geq 85 \mathrm{mmHg} \text { or } \\
\text { diagnosis for hypertension }\end{array}$ & 73 & 50 & 1.00 & 64 & 42 & 0.80 & $(0.43$, & 1.51) & 60 & 35 & 0.66 & $(0.33$ & 1.30) & 0.72 & $(0.42$ & $1.23)$ & 0.22 \\
\hline $\begin{array}{l}\text { HbA1c } \geq 5.7 \% \text { or self-reported ever physician diagnosed } \\
\text { diabetes }\end{array}$ & 90 & 30 & 1.00 & 80 & 24 & 0.90 & $(0.46$ & 1.74) & 70 & 25 & 1.08 & $(0.55$ & 2.10) & 1.06 & $(0.62$, & 1.81) & 0.83 \\
\hline Any three of the above & 109 & 14 & 1.00 & 90 & 16 & 1.43 & $(0.71$ & 2.91) & 77 & 18 & 1.53 & $(0.74$ & 3.16) & 1.38 & (0.79, & 2.43) & 0.26 \\
\hline
\end{tabular}

Any three of the above

$\begin{array}{lllll}109 & 14 & 1.00 & 90 & 16\end{array}$ 1.Metabolic syndrome is defined based on the joint interim statement of the International Diabetes Federation Task Force on Epidemiology and Prevention; National Heart, Lung, and Blood Institute; American Heart
Association; World Heart Federation; International Atherosclerosis Society; and International Association for the Study of Obesity. Analysis is based on an alternate MetS definition modified to include HbA1C instead of glucose as a marker for impaired glucose metabolism (22)

${ }^{2}$. Tertile 1 ( $\left.\mathrm{T} 1, \mathrm{nmol} / \mathrm{L}\right): \leq 17.20$ for men and $\leq 16.30$ for women; tertile 2 (T2): 17.20-22.60 for men and 16.30-21.90 for women; tertile 3 (T3): $>22.60$ for men and $>21.90$ for women. Medians (nmol/L): 13.70 for men and 14.18 for women in T1, 19.60 for men and 19.65 for women in T2, and 28.20 for men and 26.80 for women in T3

${ }^{3}$ Recent American Heart Association/National Heart, Lung, and Blood Institute guidelines for metabolic syndrome recognize an increased risk for CVD and diabetes at waist-circumference thresholds of $\geq 94 \mathrm{~cm}$ in men and $\geq 80 \mathrm{~cm}$ in women and identify these as optional cut points for individuals or populations with increased insulin resistance (22)

${ }^{4}$. ORs were adjusted for age at blood collection (years), sex, country, education (none or primary school completed, technical or professional school, secondary school, above secondary school, and not specified),

smoking status (never, former, current, and unknown), and physical activity (low, medium, high, very high, missing)

Abbreviations: MetS metabolic syndrome, $T$ tertile, OR odds ratio 
concentrations [10]. However, we did not observe an association between total neopterin concentrations and diabetes, either using the glycated haemoglobin (HbA1C), a marker of long-term blood glucose levels, or with selfreported diabetes. Similar findings have been obtained in a small saline-controlled crossover study on six healthy men (mean age 22 years) for IFN- $\gamma$ [33].

Limitations of the present study have to be taken into account. First, the mean and median concentrations of total neopterin in this study population was somewhat higher than previously reported $[2,27]$. An explanation is that our assay measures total neopterin, which is the sum of 7,8-dihydroneopterin and neopterin, in contrast to ELISA method which measures only neopterin. Nevertheless, both neopterin and total neopterin reflect inflammation and the associations between total neopterin and hsCRP, as well as the other metabolic biomarkers were comparable with previous reports (9). In addition, in our data no unexpected correlations between neopterin and basic characteristics were observed, as well as main findings were also in line with the previous reports. Secondly, the study population included controls of a nested casecontrol study; therefore, it may not be representative of the general population. However, when compared to the overall EPIC population, we have not seen major differences according to baseline characteristics, except for that our study population was slightly older, included a higher proportion of men, and a higher proportion of smokers. The range of the concentration of total neopterin reported here may not be fully representative of the general population. Thirdly, the relation between total neopterin and MetS components was assessed within the context of a cross-sectional study design, which does not allow inference about the direction of the associations. Finally, about $70 \%$ of the study participants provided non-fasting blood samples, which may have affected the TG levels; however, we have been accounting for fasting status and found essentially the same results after excluding non-fasting participants.

\section{Conclusions}

In conclusion, high total neopterin concentrations are associated with reduced HDLC, but not with overall MetS. These data support the emerging knowledge on the interplay of immune response and cholesterol metabolism. Future studies are warranted to better understand the potential role of these interrelations in chronic disease development.

\section{Methods}

\section{Study population}

The design of the EPIC cohort has been described previously [34]. In brief, EPIC recruited 518,408 volunteers from 23 centers in 10 countries (Sweden, Denmark, Norway, the
Netherlands, United Kingdom, France, Germany, Spain, Italy, and Greece) between 1992 and 2000. The eligibility criteria for participation was primarily decided within each cohort. In general, apparently healthy, middle-aged subjects who agreed to participate in the study and to have their health status followed up for the rest of their lives, were recruited. The questionnaires were completed and the blood samples were taken at recruitment.

\section{Assessment of anthropometry and lifestyle data}

The lifestyle questionnaires, which were completed by participants, included questions on diet, education, occupation, previous illnesses, alcohol, tobacco consumption, and physical activity. Informed consent forms were filled at each local center and the study was approved by the Institutional Review Board at the International Agency for Research on Cancer (IARC) and the local ethics committees. Waist circumference was measured either at the narrowest torso circumference or at the midpoint between the lower ribs and iliac crest. Systolic $\mathrm{BP}$ and diastolic BP were measured by trained personnel. Two readings were performed on the right arm in a sitting position (spaced by $1-5$ minutes) by use of a standard mercury manometer or oscillometric device. To avoid any possible white coat effect, we used the second reading, and when unavailable, the first reading.

\section{Definition of MetS}

The definition of MetS and its components have been described previously [35]. In general, we followed the harmonized definition published by Alberti et al. in 2009 [36] with slight modification in determining abnormal glucose metabolism. Briefly, MetS was defined as having any three of the following five components: 1) abdominal obesity, i.e. waist circumference is greater than or equal to $94 \mathrm{~cm}$ in men or $80 \mathrm{~cm}$ in women; 2) elevated TG, i.e. greater than or equal to $1.7 \mathrm{mmol} / \mathrm{L}$, after correction for the fasting status of the study subjects; that is, subtracting the sex-specific geometric mean difference between non-fasting and fasting subjects from the individual levels of non-fasting subjects; 3 ) reduced HDLC, i.e. less than $1.03 \mathrm{mmol} / \mathrm{L}$ in men and $1.29 \mathrm{mmol} / \mathrm{L}$ in women; 4 ) elevated BP, i.e. systolic BP $130 \mathrm{mmHg}$ or more or diastolic BP $85 \mathrm{mmHg}$ or more or self-reported physician diagnosed hypertension; and 5) abnormal glucose metabolism, i.e. self-reported physician diagnosed diabetes status or HbA1c of $5.7 \%$, which corresponds to fasting plasma glucose levels of $100 \mathrm{mg} / \mathrm{dL}$.

\section{Laboratory assays}

Plasma concentration of total neopterin (7,8-dihydroneopterin + neopterin) and PLP was determined by liquid chromatography-tandem mass spectrometry (LC-MS/MS) [37] at Bevital A/S (http://www.bevital.no), Bergen, Norway. 
Serum hsCRP was measured by a high-sensitivity assay (Beckman-Coulter, Woerden, the Netherlands), and the HDLC and TG concentrations by a colorimetric method, on a Synchron LX-20 Pro autoanalyzer (Beckman-Coulter, [38]). Measurements of HbA1c in erythrocyte hemolysate were carried out using high-performance liquid chromatography with a Bio-Rad Variant II instrument (Bio-Rad Laboratories, Hercules, California) [39]. The within and between day coefficients of variance (CV) were $3-10 \%$ for total neopterin and PLP [37]. The inter-assay CV were $6.0 \%$ and $6.5 \%$ at CRP concentrations of 1.16 and $1.89 \mathrm{mg} / \mathrm{L}$, respectively, $4.1 \%, 3.4 \%$, and $3.6 \%$ at HDLC concentrations of $0.62,1.20$, and $1.65 \mathrm{mmol} / \mathrm{L}$, respectively, and $3.3 \%, 2.1 \%$, and $2.0 \%$ at TG concentrations at 86.6, 165.9 , and $227.0 \mathrm{mg} / \mathrm{dL}$, respectively. The intra-batch CV was $2.5 \%$ for HbA1c [39].

\section{Statistical analysis}

The current analysis is based on 845 subjects ( 375 men and 470 women) who served as controls in matched case-control studies of colorectal cancer nested within the EPIC. The original aims of the nested case-control studies were to explore the risk of colon and rectal cancer in relation to MetS [35] and one-carbon metabolism biomarkers [40, 41]. MetS component measurements were not available subjects from Norway and Malmo center of Sweden. We further excluded 207 subjects who received treatment or the treatment information were missing for hyperlipidemia $(n=94)$, hypertension $(n=175)$, or diabetes $(n=15)$. Additional 44 subjects were excluded because their total neopterin measurements were not available. The final sample size for the analysis was 594.

The correlation between total neopterin and components were examined by Spearman's partial correlation coefficients (r), adjusted for age, sex, country, and smoking status. Adjusted means for total neopterin according to tertiles of each MetS component were calculated using multiple linear regression models. Because the range of the middle category of TG is narrow, we categorized TG at 1.7 and $3.4 \mathrm{mmol} / \mathrm{L}$. The dependent variable, total neopterin concentrations, was natural logtransformed and the normality assumption was tested by graphic examination of the residual distribution. The models were adjusted for age at blood collection (years), sex, country, education (none or primary school completed, technical or professional school, secondary school, above secondary school, and not specified), smoking status (never, former, current, and unknown), and physical activity (low, medium, high, very high, and missing). The adjusted means were also assessed by mutual adjustment for the other MetS components as well as PLP, due to its role in INF- $\gamma$ stimulated inflammatory responses [2, 27] and hsCRP, due to its association with low-grade inflammation. The adjusted means were then back-transformed by exponentiating the natural$\log$ transformed means from the model. The associations between total neopterin and pre-defined cutoffs of each component of MetS and the composite MetS were also examined by calculating odds ratios (OR) and $95 \%$ confidence intervals $(\mathrm{CI})$ in logistic regression analysis, adjusted for age at blood collection (years), sex, country, education (none or primary school completed, technical or professional school, secondary school, above secondary school, and not specified), smoking status (never, former, current, and unknown), and physical activity (low, medium, high, very high, missing). Total neopterin was modeled in three categories according to sex-specific tertiles. Tests for trend were performed by modelling the median values of each category as a continuous variable. Subgroup analyses were performed by age ( $<55,50-65$, and $\geq 65$ years old), sex, and body mass index (BMI, $<30$ and $\geq 30 \mathrm{~kg} / \mathrm{m}^{2}$ ).

Analyses were performed using SAS 9.3. All tests were two sided and statistical significance was assessed at the level of 0.05 .

\section{Abbreviation \\ AGM: BP: blood pressure; Cl: confidence interval; CV: coefficients of variance; EPIC: European Prospective Investigation into Cancer and Nutrition; HbA1c: glycated haemoglobin; HDLC: high-density lipoprotein cholesterol; hsCRP: high-sensitivity C-reactive protein; IFN- $\gamma$ : Interferon-gamma; MetS: metabolic syndrome; OR: odds ratio; PLP: pyridoxal 5'-phosphate; TG: Triglycerides.}

\section{Competing interests}

The authors declare that they have no competing interest.

\section{Authors' contributions}

SCC, SEV, ØM, PMU, BB, PV, and KA participated in the data analysis, manuscript writing, and interpretation of the results. BB, ML, GF, MB, RK, TK, TP, DD, ATjønneland, KO, JQ, AA, EM, MD, JMH, AB, KTK, NJW, RT,

ATrichopoulou, PL, DT, GM, CA, RT, AM, PHP, EW, RP, IL, MG, YL, AJC, ER, and $\mathrm{PV}$ were involved in data collection and interpretation of the results. All authors read and approved the final manuscript.

\section{Acknowledgements}

We thank the participants, study and administrative staff, and researchers in the European Prospective Investigation into Cancer and Nutrition (EPIC) Cohort for their outstanding cooperation.

\section{Contributors}

We would like to specifically acknowledge the contribution of Joyce Kong and Heinz Freisling (International Agency for Research on Cancer IARC-WHO, Lyon, France) for critical revision of the manuscript, and to Ellen Kohlsdorf (German Institute of Human Nutrition) and Bertrand Hemon (International Agency for Research on Cancer IARC-WHO, Lyon, France) for data management.

\section{Funding}

The EPIC cohort is supported by the Europe Against Cancer Program of the European Commission (SANCO). The individual centers also received funding from: Denmark: Danish Cancer Society; France: Ligue centre le Cancer, Institut Gustave Roussy, Mutuelle Générale de l'Education Nationale, Institut National de la Santé et de la Recherche Médicale (INSERM); Greece: the Hellenic Health Foundation, the Stavros Niarchos Foundation, and the Hellenic Ministry of Health and Social Solidarity; Germany : German Cancer Aid, and Federal Ministry of Education and Research; Italy: Italian Association 
for Research on Cancer and the National Research Council; The Netherlands: Dutch Ministry of Public Health, Welfare and Sports (WWS), Netherlands Cancer Registry (NKR), LK Research Funds, Dutch Prevention Funds, Dutch ZON (Zorg Onderzoek Nederland), World Cancer Research Fund (WCRF), Statistics Netherlands; Norway: Helga - Nordforsk centre of excellence in food, nutrition and health and The Norwegian Extra Foundation for Health and Rehabilitation, The Norwegian Cancer Society; Spain: Health Research Fund (FIS) of the Spanish Ministry of Health (Exp 96/0032, RETICC DR06/ 0020), the Spanish Regional Governments of Andalusia, Asturias, Basque Country, Murcia ( $N^{0}$ 6236), and the Navarra and the Catalan Institute of Oncology; Sweden: Swedish Cancer Society, Swedish Scientific Council, and Regional Governments of Skane and Västerbotten; UK: Cancer Research UK and Medical Research Council.

Grant supports for the biochemical measurements: HDL-C and TG were analysed with additional support from the Ministry of Public Health, Welfare and Sports, the Netherlands,German Cancer Aid, Federal Ministry for Education and Research, European Union, European Union and AIRC-ITALY, German Cancer Aid, Federal Ministry for Education and Research, European Union, Stavros Niarchos Foundation, Hellenic Ministry of Health, Hellenic Health Foundation, MRC and Cancer Research UK;Hba1c was analysed with additional support from National Cancer Institute grant 1RO1CA102460 and data analyses on CRP were performed with support from World Cancer Research Fund International and Wereld Kanker Onderzoek Fonds (WCRF NL).

The funders played no role in designing or conducting the study or in the collection, management, analysis, and interpretation of the data, nor did they have any input into the preparation, review, or approval of this manuscript.

\section{Author details}

'Institute of Population Health Sciences, National Health Research Institutes, 35 Keyan Road, Zhunan, Miaoli County 35053, Taiwan. ${ }^{2}$ Department of Epidemiology and Biostatistics, School of Public Health, Imperial College London, London, UK. ${ }^{3}$ Department of Epidemiology, German Institute of Human Nutrition Potsdam-Rehbruecke, Nuthetal, Germany. ${ }^{4}$ Department of Public Health and Primary Health Care, University of Bergen, Bergen, Norway. ${ }^{5}$ Division of Epidemiology, Norwegian Institute of Public Health, Bergen, Norway. ${ }^{6}$ Bevital AS, Bergen, Norway. ${ }^{7}$ Department of Clinical Science, University of Bergen, Bergen, Norway. ${ }^{8}$ Laboratory of Clinical Biochemistry, Haukeland University Hospital, Bergen, Norway. ${ }^{9}$ The National Institute for Public Health and the Environment (RIVM), Bilthoven, The Netherlands. ${ }^{10}$ Department of Gastroenterology and Hepatology, University Medical Centre, Utrecht, The Netherlands. "11Department of Social and Preventive Medicine, Faculty of Medicine, University of Malaya, Kuala Lumpur, Malaysia. ${ }^{12}$ Inserm, Centre for research in Epidemiology and Population Health (CESP), U1018, Nutrition, Hormones and Women's Health team, F-94805 Villejuif, France. ${ }^{13}$ University of Paris Sud, UMRS 1018, F-94805 Villejuif, France. ${ }^{14}$ IGR, F-94805, Villejuif, France. ${ }^{15}$ Division of Cancer Epidemiology, German Cancer Research Center, Heidelberg, Germany. ${ }^{16}$ Molecular Epidemiology Group, Max Delbrueck Center for Molecular Medicine (MDC), Berlin-Buch, Germany. ${ }^{17}$ Department of Epidemiology, German Institute of Human Nutrition Potsdam-Rehbrücke, Nuthetal, Germany. ${ }^{18}$ Diet, Genes and Environment, Danish Cancer Society Research Center, Copenhagen, Denmark.

${ }^{19}$ Department of Public Health, Section for Epidemiology, Aarhus University, Aarhus, Denmark. ${ }^{20}$ Public Health Directorate, Asturias, Oviedo, Spain. ${ }^{21}$ Unit of Nutrition, Environment and Cancer, Catalan Institute of Oncology-ICO, IDIBELL, L'Hospitalet de Llobregat, Barcelona, Spain. ${ }^{22}$ Escuela Andaluza de Salud Pública. Instituto de Investigación Biosanitaria de Granada (Granada.ibs), Granada, Spain. ${ }^{23}$ Consortium for Biomedical Research in Epidemiology and Public Health (CIBER Epidemiología y Salud Pública-CIBERESP), Madrid, Spain. ${ }^{24}$ Epidemiology and Health Information, Public Health Division of Gipuzkoa, Basque Regional Health Department, San Sebastian, Spain. ${ }^{25}$ Department of Epidemiology, Murcia Regional Health Council, Murcia, Spain. ${ }^{26}$ Navarre Public Health Institute, Pamplona, Spain. ${ }^{27}$ Clinical Gerontology Unit, Addenbrooke's Hospital, University of Cambridge School of Clinical Medicine, Cambridge, UK. ${ }^{28}$ MRC Epidemiology Unit, Institute of Metabolic Science, University of Cambridge School of Clinical Medicine, Cambridge, UK. ${ }^{29}$ Cancer Epidemiology Unit, Nuffield Department of Population Health, University of Oxford, Oxford, UK. ${ }^{30}$ Hellenic Health Foundation, Athens, Greece. ${ }^{31}$ Bureau of Epidemiologic Research, Academy of Athens, Athens, Greece. ${ }^{32}$ Department of Hygiene, Epidemiology and
Medical Statistics, University of Athens Medical School, Athens, Greece. ${ }^{33}$ Department of Epidemiology, Harvard School of Public Health, Boston, USA. ${ }^{34}$ Molecular and Nutritional Epidemiology Unit, Cancer Research and Prevention Institute - ISPO, Florence, Italy. ${ }^{35}$ Epidemiology and Prevention Unit, Fondazione IRCCS Istituto Nazionale dei Tumori, Milan, Italy. ${ }^{36}$ Cancer Registry and Histopathology Unit, "Civic - M.P. Arezzo" Hospital, ASP Ragusa, Italy. ${ }^{37}$ Dipartamento di Medicina Clinica e Chirurgia, Federico II University, Naples, Italy. ${ }^{38}$ Julius Center for Health Sciences and Primary Care, University Medical Center Utrecht, Utrecht, The Netherlands. ${ }^{39}$ Department of Community Medicine, Faculty of Health Sciences, University of Tromso, Tromsø, Norway. ${ }^{40}$ Department of Research, Cancer Registry of Norway, Oslo, Norway. ${ }^{41}$ Department of Medical Epidemiology and Biostatistics, Karolinska Institutet, Stockholm, Sweden. ${ }^{42}$ Samfundet Folkhälsan, Helsinki, Finland. ${ }^{43}$ Department of Medical Biosciences, Pathology, Umeå University, Umeå, Sweden. ${ }^{44}$ Department of Radiation Sciences, Oncology, Umeå University, Umeå, Sweden. ${ }^{45}$ Nutrition, Immunity and Metabolism Start-up Lab, Department of Epidemiology, German Institute of Human Nutrition Potsdam-Rehbruecke, Nuthetal, Germany.

Received: 8 December 2015 Accepted: 5 February 2016 Published online: 25 February 2016

\section{Reference}

1. Sucher R, Schroecksnadel K, Weiss G, Margreiter R, Fuchs D, Brandacher G. Neopterin, a prognostic marker in human malignancies. Cancer Lett. 2010; 287:13-22.

2. Oxenkrug G, Tucker $K L$, Requintina $P$, Summergrad P. Neopterin, a marker of interferon-gamma-inducible inflammation, correlates with pyridoxal-5'phosphate, waist circumference, hdl-cholesterol, insulin resistance and mortality risk in adult boston community dwellers of Puerto Rican origin. Am J Neuroprot Neuroregen. 2011;3:48-52.

3. Oxenkrug GF. Interferon-gamma-inducible kynurenines/pteridines inflammation cascade: implications for aging and aging-associated psychiatric and medical disorders. J Neural Transm. 2011;118:75-85.

4. Grammer TB, Fuchs D, Boehm BO, Winkelmann BR, Maerz W. Neopterin as a predictor of total and cardiovascular mortality in individuals undergoing angiography in the Ludwigshafen risk and cardiovascular health study. Clin Chem. 2009;55:1135-46.

5. Murr C, Widner B, Wirleitner B, Fuchs D. Neopterin as a marker for immune system activation. Curr Drug Metab. 2002;3:175-87.

6. Weiss G, Widner B, Zoller H, Schobersberger W, Fuchs D. Immune response and iron metabolism. Br J Anaesth. 1998;81 Suppl 1:6-9.

7. Hoffmann G, Wirleitner B, Fuchs D. Potential role of immune system activation-associated production of neopterin derivatives in humans. Inflamm Res. 2003;52:313-21.

8. Oxenkrug G. Interferon-gamma - inducible inflammation: contribution to aging and aging-associated psychiatric disorders. Aging Dis. 2011;2:474-86.

9. Capuron L, Geisler S, Kurz K, Leblhuber F, Sperner-Unterweger B, Fuchs D. Activated immune system and inflammation in healthy ageing: relevance for tryptophan and neopterin metabolism. Curr Pharm Des. 2014;20:6048-57.

10. Ledochowski M, Murr C, Widner B, Fuchs D. Association between insulin resistance, body mass and neopterin concentrations. Clin Chim Acta. 1999;282:115-23.

11. Spencer ME, Jain A, Matteini A, Beamer BA, Wang NY, Leng SX, et al. Serum levels of the immune activation marker neopterin change with age and gender and are modified by race, BMI, and percentage of body fat. J Gerontol A Biol Sci Med Sci. 2010;65:858-65.

12. Oxenkrug GF. Metabolic syndrome, age-associated neuroendocrine disorders, and dysregulation of tryptophan-kynurenine metabolism. Ann N Y Acad Sci. 2010;1199:1-14.

13. Avanzas P, Arroyo-Espliguero R, Kaski JC. Neopterin and cardiovascular disease: growing evidence for a role in patient risk stratification. Clin Chem. 2009;55:1056-7.

14. Mathis D, Shoelson SE. Immunometabolism: an emerging frontier. Nat Rev Immunol. 2011;11:81.

15. Odegaard JI, Chawla A. The immune system as a sensor of the metabolic state. Immunity. 2013;38:644-54.

16. Osborn O, Olefsky JM. The cellular and signaling networks linking the immune system and metabolism in disease. Nat Med. 2012;18:363-74. 
17. Ray KK, Morrow DA, Sabatine MS, Shui A, Rifai N, Cannon CP, et al. Long-term prognostic value of neopterin: a novel marker of monocyte activation in patients with acute coronary syndrome. Circulation. 2007:115:3071-8.

18. Thewissen MM, Damoiseaux JG, Duijvestijn AM, van Greevenbroek MM, van der Kallen CJ, Feskens EJ, et al. Abdominal fat mass is associated with adaptive immune activation: the CODAM Study. Obesity (Silver Spring). 2011;19:1690-8.

19. Kaski JC, Consuegra-Sanchez L, Fernandez-Berges DJ, Cruz-Fernandez JM, Garcia-Moll X, Marrugat J, et al. Elevated serum neopterin levels and adverse cardiac events at 6 months follow-up in Mediterranean patients with non-STsegment elevation acute coronary syndrome. Atherosclerosis. 2008;201:176-83.

20. Zangerle R, Sarcletti M, Gallati H, Reibnegger G, Wachter H, Fuchs D. Decreased plasma concentrations of HDL cholesterol in HIV-infected individuals are associated with immune activation. J Acquir Immune Defic Syndr. 1994;7:1149-56.

21. Eren E, Yilmaz N, Aydin O. High density lipoprotein and it's dysfunction. Open Biochem J. 2012;6:78-93.

22. Norata GD, Pirillo A, Ammirati E, Catapano AL. Emerging role of high density lipoproteins as a player in the immune system. Atherosclerosis. 2012;220:11-21.

23. Holven KB, Retterstol K, Ueland T, Ulven SM, Nenseter MS, Sandvik M, et al. Subjects with low plasma HDL cholesterol levels are characterized by an inflammatory and oxidative phenotype. PLoS One. 2013;8, e78241.

24. Garcia-Moll X, Cole D, Zouridakis E, Kaski JC. Increased serum neopterin: a marker of coronary artery disease activity in women. Heart. 2000;83:346-50.

25. Sulo G, Vollset SE, Nygard O, Midttun O, Ueland PM, Eussen SJ, et al. Neopterin and kynurenine-tryptophan ratio as predictors of coronary events in older adults, the Hordaland Health Study. Int J Cardiol. 2013;168(2):1435-40.

26. Ursavas A, Karadag M, Oral AY, Demirdogen E, Oral HB, Ege E. Association between serum neopterin, obesity and daytime sleepiness in patients with obstructive sleep apnea. Respir Med. 2008;102:1193-7.

27. Theofylaktopoulou D, Midttun O, Ulvik A, Ueland PM, Tell GS, Vollset SE, et al. A community-based study on determinants of circulating markers of cellular immune activation and kynurenines: the Hordaland Health Study. Clin Exp Immunol. 2013;173:121-30.

28. van Haelst PL, Liem A, van Boven AJ, Veeger NJ, van Veldhuisen DJ, Tervaert JW, et al. Usefulness of elevated neopterin and C-reactive protein levels in predicting cardiovascular events in patients with non-Q-wave myocardial infarction. Am J Cardiol. 2003;92:1201-3.

29. Avanzas P, Arroyo-Espliguero R, Cosin-Sales J, Quiles J, Zouridakis E, Kaski JC. Prognostic value of neopterin levels in treated patients with hypertension and chest pain but without obstructive coronary artery disease. Am J Cardiol. 2004:93:627-9.

30. Avanzas P, Arroyo-Espliguero R, Quiles J, Roy D, Kaski JC. Elevated serum neopterin predicts future adverse cardiac events in patients with chronic stable angina pectoris. Eur Heart J. 2005;26:457-63.

31. Pacileo M, Cirillo P, De RS, Ucci G, Petrillo G, Musto DS, et al. The role of neopterin in cardiovascular disease. Monaldi Arch Chest Dis. 2007;68:68-73.

32. Schennach H, Murr C, Gachter E, Mayersbach P, Schonitzer D, Fuchs D. Factors influencing serum neopterin concentrations in a population of blood donors. Clin Chem. 2002:48:643-5.

33. de Metz J, Sprangers F, Endert E, Ackermans MT, ten Berge IJ, Sauerwein HP, et al. Interferon-gamma has immunomodulatory effects with minor endocrine and metabolic effects in humans. J Appl Physiol. 1999:86:517-22.

34. Riboli E, Hunt KJ, Slimani N, Ferrari P, Norat T, Fahey M, et al. European Prospective Investigation into Cancer and Nutrition (EPIC): study populations and data collection. Public Health Nutr. 2002;5:1113-24.

35. Aleksandrova $K$, Boeing $H$, Jenab M, Bas Bueno-de-Mesquita $H$, Jansen $E$, van Duijnhoven FJ, et al. Metabolic syndrome and risks of colon and rectal cancer: the European prospective investigation into cancer and nutrition study. Cancer Prev Res (Phila). 2011:4:1873-83.

36. Alberti KG, Eckel RH, Grundy SM, Zimmet PZ, Cleeman JI, Donato KA, et al. Harmonizing the metabolic syndrome: a joint interim statement of the International Diabetes Federation Task Force on Epidemiology and Prevention; National Heart, Lung, and Blood Institute; American Heart Association; World Heart Federation; International Atherosclerosis Society; and International Association for the Study of Obesity. Circulation. 2009;120:1640-5.
37. Midttun O, Hustad S, Ueland PM. Quantitative profiling of biomarkers related to B-vitamin status, tryptophan metabolism and inflammation in human plasma by liquid chromatography/tandem mass spectrometry. Rapid Commun Mass Spectrom. 2009:23:1371-9.

38. van Duijnhoven FJ, Bueno-de-Mesquita HB, Calligaro M, Jenab M, Pischon T, Jansen EH, et al. Blood lipid and lipoprotein concentrations and colorectal cancer risk in the European prospective investigation into cancer and nutrition. Gut. 2011;60:1094-102.

39. Rinaldi S, Rohrmann S, Jenab M, Biessy C, Sieri S, Palli D, et al. Glycosylated hemoglobin and risk of colorectal cancer in men and women, the European prospective investigation into cancer and nutrition. Cancer Epidemiol Biomarkers Prev. 2008;17:3108-15.

40. Eussen SJ, Vollset SE, Igland J, Meyer K, Fredriksen A, Ueland PM, et al. Plasma folate, related genetic variants, and colorectal cancer risk in EPIC. Cancer Epidemiol Biomarkers Prev. 2010;19:1328-40.

41. Eussen SJ, Vollset SE, Hustad S, Midttun O, Meyer K, Fredriksen A, et al. Plasma vitamins B2, B6, and B12, and related genetic variants as predictors of colorectal cancer risk. Cancer Epidemiol Biomarkers Prev. 2010;19:2549-61.

\section{Submit your next manuscript to BioMed Central and we will help you at every step:}

- We accept pre-submission inquiries

- Our selector tool helps you to find the most relevant journal

- We provide round the clock customer support

- Convenient online submission

- Thorough peer review

- Inclusion in PubMed and all major indexing services

- Maximum visibility for your research

Submit your manuscript at www.biomedcentral.com/submit
Ciomed Central 